\title{
Old age psychiatry and geriatric medicine: shared challenges, shared solutions?
}

\author{
James Fisher and Andrew Teodorczuk
}

\section{Summary}

The co-occurrence of physical and mental ill health means there is considerable overlap between the patients that geriatric medicine and old age psychiatry serve. In this editorial we detail similarities between the specialisms, highlight the common challenges facing them and argue that closer alignment holds the potential to improve patient care.

\author{
Declaration of interest \\ None.
}

\section{Copyright and usage}

(c) The Royal College of Psychiatrists 2017.
James Fisher (pictured) is a consultant geriatrician working at Northumbria Healthcare NHS Foundation Trust and as an Associate Clinical Lecturer at Newcastle University. Andrew Teodorczuk previously worked as a consultant old age psychiatrist at Northumberland, Tyne and Wear NHS Foundation Trust and as an honorary senior lecturer at Newcastle University; he is currently associate professor in medical education and an old age psychiatrist at Griffith University, Australia.

\section{Different specialties, similar challenges}

In the UK, old age psychiatry and geriatrics are notable for the fact that their consultant workforces are comparatively thinly spread. Psychiatry is the fifth largest specialty, representing $10.5 \%$ of the consultant workforce, however, only $15 \%$ of consultants specialise in old age psychiatry. ${ }^{1}$ Currently, there are 1.1 full-time equivalent (FTE) old age psychiatry consultants per 100000 population, ${ }^{1}$ despite the Department of Health's recommendation being 1.8 per 100000 . By comparison, working-age adult psychiatry has 4.1 FTE consultants per 100000 . Numerically, geriatric medicine is the largest hospital medical specialty, ${ }^{2}$ with approximately 2.0 FTE per 100000 population. The British Geriatrics Society recommends a minimum of 1 FTE geriatrician per 50000 ; although on average this ratio is met nationally, there is considerable UK variation (1:46000-1:86000). ${ }^{3}$ Given the ageing population and expanding workload, workforce planning in both specialties remains precarious.

In old age psychiatry, estimates suggest a $45 \%$ increase in consultant numbers is required by $2033,{ }^{1}$ yet Centre for Workforce Intelligence data predict a decrease of $7 \%$ by 2033 . Similarly, in geriatric medicine, it is recognised that the changing population demographic necessitates vast expansion in consultant numbers. In sum, both specialities are essential to the future attainment of high-quality care that meets the demands of the ageing population, yet both face huge workforce challenges if this is to be achieved.

Both specialties also face an identity crisis. Geriatric medicine has, for some time, debated what to call itself; this may in part account for a degree of confusion among the general population as to what a geriatrician does. ${ }^{4}$ Similar challenges exist in old age psychiatry, where it is unclear to the public what psychiatrists do, let alone old age psychiatrists. It is also recognised that medical students do not entirely understand the psychiatrist's role. ${ }^{5}$ This confusion may be compounded by the fact that in Europe, it is not uncommon for neurologists to fulfil old age psychiatry duties.
Consequently, old age psychiatry as a specialty is seen by some as under threat, even though the role of the old age psychiatrist, and their contribution to the care of older people, has never been in greater demand.

Of huge concern for both specialties are looming recruitment challenges. Negative attitudes towards geriatric medicine exist among medical students, with some perceiving the specialty to lack prestige. ${ }^{6}$ Alarmingly, some junior doctors ${ }^{7}$ and medical students ${ }^{8}$ exhibit negative attitudes towards caring for cognitively impaired patients. These attitudes may be compounded by the comparative lack of exposure to both specialties at undergraduate level; in the UK, the median total time spent on undergraduate teaching in ageing and geriatric medicine was only 55.5 hours. ${ }^{9}$ Short rotational attachments restrict opportunities to see benefits in patients with chronic illnesses and may reinforce the sense of 'therapeutic nihilism' held by some. ${ }^{7}$ Countering negative attitudes that are shaped by societal ageism is challenging but essential. The sooner this is addressed the greater the likely benefit; as such, as a starting point, we would advocate that students at undergraduate level reflect on society's, and their own, attitudes and are exposed to teaching from patients and carers at all stages of the curriculum. ${ }^{10}$

Old age psychiatry recruitment is further complicated by considerable attrition of doctors after core training; nearly one in five does not progress to higher training. ${ }^{1}$ The reasons for this are poorly understood, but difficulty passing the required examinations to achieve membership of the Royal College of Psychiatrists may be a factor. There are also issues at the same transition point within general medical training. The role of the medical registrar (the senior training grade for future hospital medical consultants), has been highlighted as a potential deterrent to undertaking higher specialty training in specialties including acute medical work, such as geriatric medicine. ${ }^{7}$

\section{Shared solutions?}

Although these challenges make sober reading, opportunities do exist for both specialties. First, we outline 'top-down' interventions designed to address these challenges; second, an example of a 'grass-roots' initiative will be shared.

In 2014 Health Education England announced their aim to increase the number of foundation doctors with psychiatry posts from $2-4 \%$ to $22.5 \%,{ }^{11}$ since foundation doctors who undertook psychiatry posts were more likely to choose psychiatry as a career than those that did not. ${ }^{12}$ Financial incentives are also being 
considered; the proposed new contract for UK junior doctors includes a pay premium for those entering psychiatry training. A 2013 national survey of core medical trainees highlighted dissatisfaction with training. To address this, a set of quality criteria were developed, including a drive for all core medical trainees to undertake a geriatric medicine placement. This may promote recruitment to geriatric medicine, since it is recognised that doctors opting to become geriatricians, tended to decide at a later stage in their training. ${ }^{13}$ The recent Shape of Training (SoT) report proposes fundamentally restructuring UK postgraduate medical training. ${ }^{14}$ With SoT, post-foundation programme doctors will enter 'broad-based' specialty training, where specialties are grouped into 'patient care themes' defined by 'the dynamic and interconnected relationships between the specialties.' ${ }^{14}$ Geriatric medicine and old age psychiatry would make natural partners in this system. The aspiration is that enabling doctors to train across a breadth of similar specialties will offer greater career flexibility within care themes. The proposals will reduce the duration of training; hence concern that future doctors will not be able to achieve the same level of expertise as current specialists. In summary, there is policy momentum to address the challenges outlined above - time will tell if this achieves the desired impact on the workforce.

'Top-down' change alone however, is unlikely to succeed without 'grass-roots' momentum. An excellent example of an 'organic' initiative to encourage specialty recruitment, is the Association for Elderly Medicine Education (AEME). ${ }^{7}$ AEME was founded by a group of geriatric medicine registrars in Northeast England in 2012, who aimed to encourage interest and recruitment into their specialty. A multifaceted approach was employed that included face-to-face conferences (Geriatrics for Juniors (G4J)) and e-learning. The first G4J event attracted over 100 UK junior doctors - the popularity was such that the event is now annual and is complemented by a series of satellite, local events (G4J Connect). Data from the initial G4J demonstrated a positive impact on delegates' perceptions about the medical registrar role. ${ }^{7}$ AEME have also developed a series of older-person-specific e-learning resources - Mini Geriatric e-learning modules (Mini-GEMs). Mini-GEMs are video podcasts that offer concise education about commonly encountered, olderperson-specific clinical problems (http://aeme.org.uk/mini-gems). AEME's projects unearthed huge appetite and enthusiasm among junior doctors for geriatric medicine and highlighted an unmet educational need. Despite their relative lack of seniority, junior doctors possess a unique insight into their training - this, along with their innate enthusiasm, can be harnessed to inform recruitment initiatives. Similar initiatives in old age psychiatry are welcomed.

\section{A synthesis of specialties?}

We argue that tackling the unprecedented challenges facing both specialties will require bold and innovative action. Given the commonalities between specialties, we propose closer integration of the two specialties, in terms of both service provision and training. With regard to provision of psychiatry services, we note with concern increasingly blurred roles, with old age psychiatry working with general psychiatry in so-called 'ageless' services. It has been suggested that moving towards such models of service delivery has had a detrimental effect on quality of care, patient safety, service efficiency and staff morale. ${ }^{15}$ We challenge the 'ageless' services configuration, asserting that the care of complex, older patients is a subspecialty in its own right and thus services ought to be arranged accordingly.
Recent research supports this assertion; a study comparing the quality of care of people with mental illness who grow old and transfer to an older adults service, with those who remain in general adult services, found that old age psychiatry services were better placed to meet the needs of older people with mental illness. ${ }^{16}$ Arguably, the old age psychiatry patient group is more akin to that served by geriatric medicine, yet further problems may result if service configuration inadvertently introduces 'competition' for similar patients between specialisms. In some areas for example, separate memory clinics are provided by both physicians and psychiatrists. This may not be the most effective use of resources, may produce unhelpful interspecialty rivalry and may compound confusion regarding the roles of each specialty. More coordinated, joined-up working between specialties is critical.

The idea of 'reciprocal training' between our specialties is not new. At present, however, there is no formalised inclusion of geriatric medicine competencies within the old age psychiatry specialty training curriculum. Some trainees opt to undertake a diploma in geriatric medicine, but uptake is not widespread. The current curriculum for training in geriatric medicine does incorporate some old age psychiatry competencies. 'Low-level' competencies sit within 'core' curriculum and are mandatory; 'higher-level' competencies are optional and completion of these is the exception, rather than the norm. It is recognised that some old age psychiatry trainees feel their medical knowledge is lacking and that some junior doctors cite a lack of 'medical' work as a deterrent to undertaking psychiatry training. Conversely, complex old age psychiatry competencies are recognised as a 'blind spot' for many geriatric medicine trainees, and a long-term commitment to being the medical registrar is, for some, a deterrent.

To remedy these issues, we propose a new, shared training pathway that funnels trainees into a new subspecialty, geripsychiatry (in practice, not just name) where experience is gained in both domains. We suspect the marriage of experience within geriatric medicine and old age psychiatry environments would be attractive to many junior doctors. This hybrid specialist training route would produce clinicians with a unique skill set, well suited to the complexities of medical and psychiatric care and more attuned to the increasingly important medicolegal context within which care of older people is delivered. SoT provides an outline for the evolution of such a training pathway, but constricted training time may prove a barrier to integration. Currently, a lack of joined-up training trickles downstream and results in a lack of joined-up care. We call for the medical Royal Colleges, the professional bodies responsible for old age psychiatry and geriatric medicine training, and Health Education England to reset the training compass and to determine how innovative approaches to training in these fields might be developed.

James Fisher, MD, DipClinEd, MClinRes, MRCP, MBBS, Department of Geriatric Medicine, Northumbria Healthcare NHS Foundation Trust, UK; Andrew Teodorczuk MD, DipClinEd, MBChB(Edinb), MRCPsych, School of Medicine, Griffith University, Queensland, Southport, Australia

Correspondence: James Fisher, Northumbria Healthcare NHS Foundation Trus North Tyneside General Hospital, Rake Lane, North Shields NE29 8NH, UK. Email: drjamesfisher@hotmail.com

First received 7 May 2016, final revision 29 Sep 2016, accepted 10 Nov 2016

\section{References}

1 Centre for Workforce Intelligence. In-Depth Review of the Psychiatrist Workforce. Centre for Workforce Intelligence, 2014 (http://www.cfwi.org.uk/ publications/in-depth-review-of-the-psychiatrist-workforce/).

2 Gordon H. Census of Consultant Physicians and Higher Specialty Trainees in the UK, 2013-14: Data and Commentary. Federation of the Royal Colleges 
of Physicians of the UK, 2015 (https://www.rcplondon.ac.uk/file/1551/ download?token $=$ DaOVmvTz)

3 Royal College of Physicians. Consultant Physicians Working with Patients The Duties, Responsibilities and Practice of Physicians in Medicine. Royal College of Physicians, 2011

4 Campbell JY, Durso SC, Brandt LE, Finucane TE, Abadir PM. The unknown profession: a geriatrician. J Am Geriatr Soc 2013; 61: 447-9.

5 Curtis-Barton MT, Eagles JM. Factors that discourage medical students from pursuing a career in psychiatry. Psychiatrist 2011; 35: 425-9.

6 Robbins TD, Crocker-Buque T, Forrester-Paton C, Cantlay A, Gladman JRF, Gordon AL. Geriatrics is rewarding but lacks earning potential and prestige: responses from the national medical student survey of attitudes to and perceptions of geriatric medicine. Age Ageing 2011; 40: 405-8.

7 Fisher JM, Hunt K, Garside MJ. Geriatrics for juniors: tomorrow's geriatricians or another lost tribe? J R Coll Physicians Edinb 2014; 44: 106-10.

8 Cutler J, Alspector S, Harding K, Wright L, Graham M. Medical students' perceptions of psychiatry as a career choice. Acad Psychiatry 2006; 30 : 144-9.

9 Gordon AL, Blundell A, Dhesi JK, Forrester-Paton C, Forrester-Paton J, Mitchell HK, et al. UK medical teaching about ageing is improving but there is still work to be done: the second national survey of undergraduate teaching in ageing and geriatric medicine. Age Ageing 2014; 43: 293-7.
10 Tullo E, Khoo TK, Teodorczuk A. Preparing to meet the needs of an ageing population - a challenge to medical educators globally. Med Teach 2015; $\mathbf{3 7}$ $105-7$.

11 Health Education England. Broadening the Foundation Programme. Health Education England, 2014 (https://www.hee.nhs.uk/sites/default/files/ documents/Broadening\%20the\%20foundation\%20report.pdf).

12 Kelley TA, Brown J, Carney S. Foundation Programme psychiatry placement and doctors' decision to pursue a career in psychiatry. Psychiatrist 2013; 37: $30-2$.

13 Briggs S, Atkins R, Playfer J, Corrado OJ. Why do doctors choose a career in geriatric medicine? Clin Med 2006; 6: 469-72.

14 Shape of Training. Securing the Future of Excellent Patient Care: Final Report of the Independent Review Led by Professor David Greenaway. Shape of Training, 2013 (http://www.shapeoftraining.co.uk/reviewsofar/1788.asp).

15 Warner JP. Old age psychiatry in the modern age. Br J Psychiatry 2015; 207: $375-6$.

16 Abdul-Hamid WK, Lewis-Cole K, Holloway F, Silverman AM. Comparison of how old age psychiatry and general adult psychiatry services meet the needs of elderly people with functional mental illness: cross-sectional survey. Br J Psychiatry 2015; 207: 440-3. 